\title{
Research on Innovation Performance in Multi-level Collaborative Innovation Network
}

\author{
Jing Li, Huimin Tang*, Haibin Tang \\ Xinhua College of Sun Yat-sen University \\ Guangzhou, 510520
}

\begin{abstract}
Taking the patent data of Guangzhou bio pharmaceutical industry in 2017 as an example, this paper constructs a double-level collaborative innovation network, which consists of the $R \& D$ institution network and the $R \& D$ staff network. Exponential Random Graph Models is used to analyze the present collaborative innovation situation of the constructed network. Factors that affect $R$ \& D staff's innovation performance are tested. We find that the centrality nodes in $\mathbf{R} \&$ $D$ staff network has a significant positive effect on their innovation performance, and the clustering coefficient of the nodes has a significant negative impact on innovation performance. The centrality of $R \& D$ institutions can moderate the effect of $R \& D$ staff's centrality and clustering coefficient on innovation performance. The number of structural holes of $R \&$ $D$ institutions can also moderate the impact of $R \& D$ staff's clustering coefficient on innovation performance.
\end{abstract}

keywords-multi-level network; synergetic innovation; ERGM; innovation performance

\section{INTRODUCTION}

China proposes clearly that innovation is the first driving force for development and the strategic support for building a modern economic system, which points out that it is necessary to deepen the reform of the science and technology system and establish an innovative system of deep integration of industry, academia and research. Due to the limited nature of resources and knowledge technology, collaborative innovation between organizations has become one of the major technological innovation models. The organizational form of collaborative innovation is a collaborative innovation network, which helps to reduce the uncertainty of the innovation environment, and produces synergies of " $1+1>2$ " to achieve technological innovation and technological advancement [1]. There are some studies at home and abroad on the relationship between collaborative innovation networks and innovation performance.
Chaoyong Tang and Chongxin Niu (2017) found that the collaborative innovation network has a significant positive impact on innovation performance[2]based on the questionnaire survey data of 168 technology-based enterprises in Taiyuan City. A Ruan, J Chen (2017) explored the influence of network structure variables such as centrality and structural holes and tacit knowledge on the innovation performance of 161 enterprises [3], pointing out that high network centrality is conducive to fostering high innovation performance. J Peng et al. (2014) constructed a network of 149 employees and found that the centrality of network nodes has a significant positive impact on employee innovation performance [4]. The existing research on enterprise innovation performance and employee innovation performance only analyzes the characteristics of collaborative innovation network from one level, and the relationship between network structure characteristic variables and innovation performance. In recent years, multi-level networks have been analyzed in the field of social network analysis.

The starting point of this new method is that the nodes in the network have subordination to the organization. Such relationships make networks with different levels, such as organizational and individual levels. Figure1 is a schematic diagram of a multi-level R\&D network. The two network levels are mutually influential and change together. For example, the organizational innovation atmosphere will affect employees' willingness to innovate to a certain extent, which will affect their innovation performance [5]. There is little research on how multi-level composite network structures affect individual innovation performance in collaborative innovation networks. This paper takes patent data as an example to build a two-tier collaborative innovation network for R\&D institutions and R\&D personnel, and explores the impact of network structure on $R \& D$ personnel innovation performance

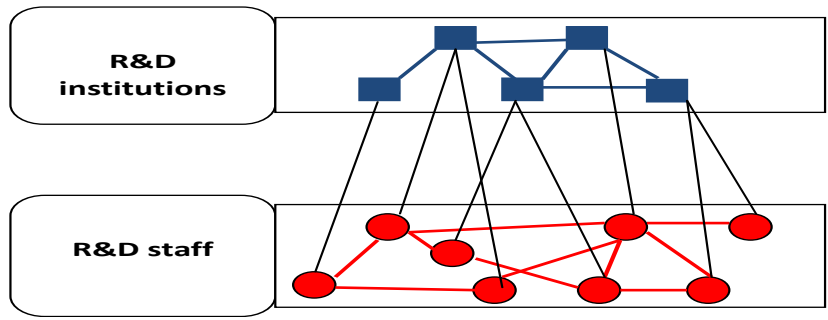

Fig. 1 Multi-level network diagram

CLC Number: F 273.1. 


\section{MULTI-LEVEL COLLABORATIVE INNOVATION NETWORK}

\section{A. Construction of Multi-level collaborative innovation} network

Patent is one of the main achievements of scientific and technological innovation, it is often used as a measure of innovation level and a construction material of innovation network in domestic and foreign research $[6,7]$. To retrieve the common patent data of Guangzhou biomedical field in 2017 from the China Knowledge Network patent database, the paper uses this as an example to construct a multi-level collaborative innovation network, which consists of a collaborative innovation network between patent inventors and a network of cooperation between patent applicants. The patent inventor belongs to a research and development institution and combines the two networks to form a multi-level network. The multi-level collaborative innovation network constructed in this paper includes $26 \mathrm{R} \& \mathrm{D}$ institutions and $177 \mathrm{R} \& \mathrm{D}$ personnel. The two levels of networks are shown in Figures 2 and 3 respectively. The square in Figure 2 represents the R\&D organization. If two R\&D institutions jointly apply for a patent in 2017, the two institutions are connected by one side. The dots in Figure 3 indicate the R\&D staff. The connection between the two R\&D personnel indicates that the two people participated in the invention of a patent. Combining the R\&D network with the R\&D network, the overall collaborative innovation network shown in Figure 4 is obtained (square represents the R\&D organization, and the circle represents the R\&D staff). This overall network reflects both the subordinate relationship between $R \& D$ institutions and $R \& D$ personnel, also the synergy between R\&D institutions and R\&D personnel.

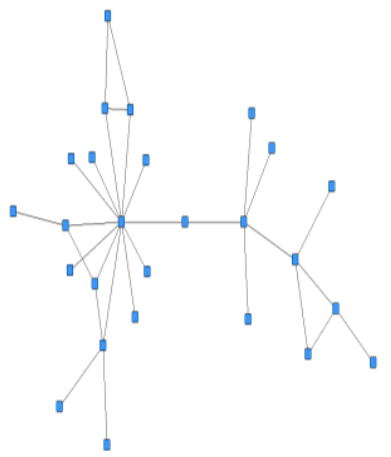

Fig.2. R\&D Inter-agency Network

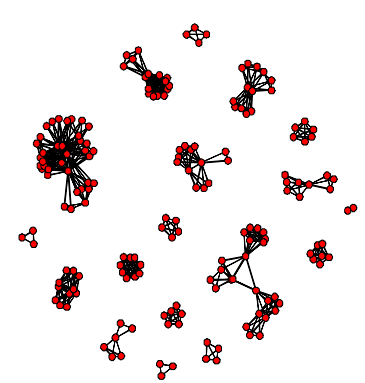

Fig.3. R\&D staff network

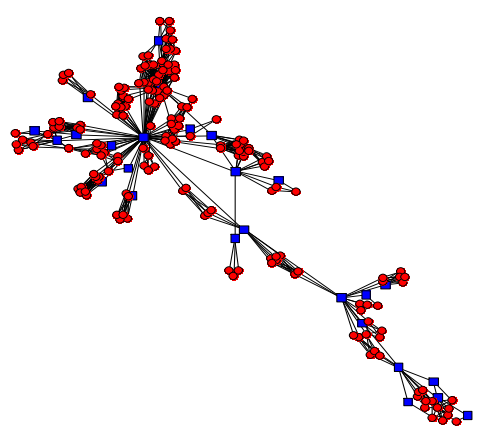

Fig.4. Multi-level collaborative innovation network

\section{B. Analysis Network Structure}

This section uses the new method of social network analysis--- Exponential Random Graph Models, to analyze the two constructed networks to clarify the status of collaborative innovation between the two networks. The exponential random graph model is mainly used to discover network structures that have a significant effect on network formation. The general form is: $P(X=x)=(1 / k) \exp \left\{\sum \theta_{A} Z_{A}(x)\right\}$, the $\mathrm{K}$ is a constant, ensuring the network structure $\mathrm{x}$ is between 0 and 1 , and $\theta_{\mathrm{A}}$ is the corresponding network structure statistics. The coefficient of the quantity $\mathrm{Z}_{\mathrm{A}(\mathrm{x})}$ [8]. Table 1 lists several network structures and their parameter estimates. The parameter estimation is implemented by the Markov Chain Monte Carlo Estimation (MCMC) using the statnet package in the $\mathrm{R}$ language, using Markov chain Monte Carlo estimation (MCMC)to implement.

TABLE I NETWORK STRUCTURE AND PARAMETER ESTIMATION

\begin{tabular}{|c|c|c|c|c|}
\hline Network structure & Schematic & description & R\&D network & R\&D staff network \\
\hline Edges & -0 & $\begin{array}{l}\text { Trends of } \begin{array}{r}\text { Basic } \\
\text { cooperative research } \\
\text { and development }\end{array}\end{array}$ & $-2.3413 * * *$ & $-3.3347 * * *$ \\
\hline Second path & & Transitive trend & $-0.3429 * * *$ & $-0.21102 * * *$ \\
\hline Three stars & & Center-periphery & $-0.10405 * * *$ & $-0.03765^{* * * *}$ \\
\hline alt-triangle triangle & & $\begin{array}{l}\text { Condensed subgroup } \\
\text { trend }\end{array}$ & & 3.1885 *** \\
\hline
\end{tabular}

It can be seen from Table 1 that the coefficients of the edge structures of the two networks are both negative, indicating the network density is low [7], the overall coordination between the network nodes is not high. The coefficient of the two-path structure embodying transitivity is also negative, indicating that there is still much room for improvement in the linkage level 
between the R\&D institutions and the R\&D personnel. Taking the R\&D network as an example, the coefficient -0.3429 indicates that the transitive effect of the two-path structure in the network can be quantified as $\exp (-2.341 \quad 3-0.342$ $9) /(1+\exp (-2.3413-0.3429))=0.064$ The probability that the two-path structure affects the establishment of a new edge in the R\&D network is $6.4 \%$. Similarly, the transitive effect of the two-path structure in the R\&D network can be quantified as $\exp (-3.3347-0.21102) /(1+\exp (-3.334 \quad 7-0.21102))=0.028$, the two-path structure affects the network. The probability of a collaborative innovation relationship between the two R\&D personnel is only $2.8 \%$. The coefficient of the Samsung structure that reflects the center-edge trend in the two networks is significant, indicating that there are core R\&D nodes in the network. However, the negative coefficient indicates that the leading, driving and radiating effects of these core nodes are still relatively low. In the R\&D network, the driving effect of the core node can be quantified as $\exp (-2.341 \quad 3-0.104$ $05) /(1+\exp (-2.3413-0.10405))=0.079$, that is, the core $R \& D$ organization drives the network.

The probability of establishing a collaborative innovation relationship between the two $\mathrm{R} \& \mathrm{D}$ institutions in the network is $7.9 \%$. Similarly, the driving effect of the core nodes in the R\&D network can be quantified as $\exp (-3.334 \quad 7-0.037$ $65) /(1+\exp (-3.3347-0.03765))=0.033$, that is, the core $R \& D$ personnel drive the two in the network. The probability of $R \& D$ personnel establishing a cooperative $R \& D$ relationship is $3.3 \%$. The leading radiation effects of the core nodes of the two networks can still be greatly improved. The coefficient of the interactive triangle structure in the R\&D network is significant, indicating that there are obvious sub-groups in the network, which means that the local coordination is very close. This can be confirmed from the network of developers in Figure 3. The R\&D network is actually composed of multiple $R \& D$ subgroups.

\section{INNOVATION PERFORMANCE IN MULTI-LEVEL COLLABORATIVE INNOVATION NETWORK}

\section{A. Research hypothesis}

In a collaborative innovation network, the nodes of it has different opportunities to acquire knowledge because of their different locations. The location of a network node can be measured by its centrality. Some studies have found that the centrality of network nodes can significantly affect the innovative performance of nodes [3, 4, 9]. In the collaborative innovation network of R\&D personnel, highly-centered $R \& D$ personnel can obtain a large amount of professional information and knowledge, including heterogeneous and complementary knowledge [4]. It is conducive to enriching and perfecting its own knowledge structure, finding suitable R\&D partners, improving innovation output. Therefore, this article proposes:

Hypothesis 1: The centrality of $R \& D$ personnel is positively affects their innovation performance.

The clustering coefficient of a network node measures the degree of interconnection between neighbor nodes of the node. The high clustering coefficient of a node in the network, meaning that the neighboring nodes of the node are also closely connected. Thus facilitates the transfer of information in the cluster, but it may also cause assimilation in the cluster, resulting in homogeneity in the knowledge structure of the cluster members. Bringing a lot of repeated information [10], which is not conducive to the innovation of developers. Because heterogeneous knowledge plays a crucial role in the innovation process [11]. Therefore, this article proposes:

Hypothesis 2: The clustering coefficient of R\&D personnel negatively affects their innovation performance.

The structural characteristics of collaborative innovation networks are closely related to the innovation performance of network nodes [12]. In a multi-level collaborative innovation network, the knowledge organization of the R\&D personnel is not only related to the location of the network of personnel, but also to the total knowledge of the R\&D institutions it belongs to. It means the interaction between R\&D personnel and their environment will affect their innovation to some extent [13].

Therefore, when investigating the innovation performance of R\&D personnel, it is necessary to embed $R \& D$ personnel in their R\&D institutions. Organizations that are centrally located in the R\&D network tend to have more accumulation of knowledge, better R\&D results than other institutions [14]. Thanks to this, employees with high-centred $R \& D$ institutions may also have easier access to critical knowledge from within, resulting in better innovation. In addition, the large amount of knowledge of R\&D institutions also means the diversity of knowledge within the organization. This can weaken the knowledge homogeneity of local clustering in the network to a certain extent. Therefore, this article proposes:

Hypothesis 3: The centrality of R\&D institutions moderates the impact of the centrality of their R\&D staff on innovation performance.

Hypothesis 4: The centrality of the R\&D institution moderates the impact of the clustering coefficient of its R\&D node on innovation performance.

A structural hole is an important one in a network and refers to a non-redundant connection between two nodes. The existence of structural holes means that the third node can act as an intermediary, which is beneficial to the formation of weak connections and bridge connections between R\&D institutions [15]. Tight network connections can improve communication between R\&D institutions, but it may lead to the homogenization of knowledge, and the inter-node structural holes can break the negative impact of this over-embedding, benefiting the creative ideas [16]. Burt's classical theory points out that structural holes are beneficial to bridge nodes to obtain the flow of information and knowledge between other nodes, which is in a dominant position in the network [17]. R\&D institutions with more structural holes are subject to fewer external restrictions and are more flexible [18]. The employees of these institutions also enjoy more autonomy. Combined with the rich variety of knowledge and information brought about by the bridges of institutions, employees of these organizations may have better innovation performance. Therefore, this article proposes:

Hypothesis 5: The structural hole position in R\&D institutions moderates the impact of the centrality of their 
R\&D staff on innovation performance.

Hypothesis 6: The structural hole position in R\&D institutions moderates the impact of the R\&D staff's clustering coefficient on innovation performance.

\section{B. Hypothetical Test}

This article uses $177 \mathrm{R} \& \mathrm{D}$ personnel's patents obtained in 2017 (including proprietary patents and joint patents) as a measure of their innovation performance. The centrality of R\&D institutions and R\&D personnel is indicated in terms of degree centrality. The degree of centrality of a node is the number of nodes directly connected to the node, which is the most intuitive indicator. The structural hole position in R\&D institutions is calculated based on the limit index [10]. The results of correlation analysis of each variable are shown in Table 2

TABLE II RELATED ANALYSIS

\begin{tabular}{|c|c|c|c|c|c|c|}
\hline variable & 1 & 2 & 3 & 4 & 5 & VIF \\
\hline 1.Innovation performance & 1 & & & & & \\
\hline $\begin{array}{l}\text { 2.personnel } \\
\text { centerality }\end{array}$ & $.551 * *$ & 1 & & & & 1.584 \\
\hline 3.Personnel clustering coefficient & $-.559 * *$ & $-.551 * *$ & 1 & & & 1.666 \\
\hline 4.Institutional centrality & 0.078 & $.182 *$ & $-.152 *$ & 1 & & 1.038 \\
\hline $\begin{array}{l}\text { 5. Number of institutional } \\
\text { structures }\end{array}$ & $-.217 * *$ & .089 & $.256^{* *}$ & -.003 & 1 & 1.165 \\
\hline
\end{tabular}

$$
\text { Note: } * \mathrm{p}<0.05 ; * * \mathrm{p}<0.01 ; * * * \mathrm{p}<0.001
$$

It can be seen from Table 2 that the correlation coefficients between the respective variables are all below 0.6 , the variance inflation factor (VIF) is less than 10, there is no obvious multicollinearity, which can be further analyzed. Since the dependent variable is the number of patents obtained by the $\mathrm{R} \& \mathrm{D}$ personnel and belongs to the non-negative counting variable, the negative binomial regression model is used for parameter estimation. The results are shown in Table 3.

TABLE III

PARAMETER ESTIMATION AND HYPOTHESIS TEST

\begin{tabular}{llllll}
\hline & Model 1 & Model 2 & Model 3 & Model 4 & Model 5 \\
\hline constant & $2.751^{* * *}$ & $2.611^{* * *}$ & $1.199 * * *$ & $1.187 * * *$ & $1.316^{* * *}$ \\
Personnel centerality & $7.714^{* *}$ & $6.002^{*}$ & $8.1619^{* *}$ & $7.5121^{*}$ & $5.483^{*}$ \\
Personnel clustering coefficient & $-2.033^{* * *}$ & $-1.984^{* * *}$ & $-2.1351 * * *$ & $-2.5948^{* * *}$ & $-4.641^{* * *}$ \\
Institutional centrality & & 0.173 & 0.176 & 0.635 & 0.705 \\
Structural holes & & & &
\end{tabular}

Personnel centrality $X$

$1.603^{*}$

Institutional centerality

Personnel clustering coefficient

$\times$

Institutional centerality

Personnel centralit $X$

Structural holes

Personnel clustering coefficient

Structural holes

AIC

Log lik.

803.17

$-795.17$
$3.0018 *$

$5.957^{* * *}$

$\begin{array}{lll}803.83 & 806.09 & 786.44\end{array}$

\begin{tabular}{lll}
-791.835 & -794.09 & -774.445 \\
\hline
\end{tabular}

As can be seen from Table 3, in Model 1, the degree of centrality of the $R \& D$ personnel has a significant positive impact on its innovation performance, so Hypothesis 1 holds. The clustering coefficient of the R\&D node has a significant negative impact on its innovation performance, and Hypothesis 2 holds. Model 2 and Model 3 are used to test whether the centrality of the R\&D institution can moderate the influence of the centrality of the $R \& D$ personnel ,and the clustering coefficient on the innovation performance. The interaction coefficient of Model 2 and Model 3 are both significant, and the moderating effect is obvious. so, the assumption of 3 is valid and Hypothesis 4 is valid. Model 4 and Model 5 test whether the structural hole position in the R\&D organization can moderate the influence of the centrality of the $R \& D$ personnel and the clustering coefficient on the innovation performance.

The coefficient of the interaction term in Model 4 is not significant $(\beta=4.653, p=0.729)$, that is, the structural hole position of the R\&D institution cannot significantly moderate the influence of the centrality for the $R \& D$ personnel on its innovation performance, so the hypothesis 5 does not hold. The coefficient of the interaction term in Model 5 is significant, and the structural hole position of the organization can moderate the influence of the clustering coefficient of the $R \& D$ personnel on the innovation performance, so the hypothesis 6 holds.

\section{Discussion}

This paper verifies the centrality of R\&D personnel can affect their innovation performance significantly. This is consistent with the conclusions of some existing studies [3, 4, 9]. On this basis, we further discovered that the influence of R\&D personnel's centrality on innovation performance in a multi-level collaborative innovation network is regulated by the centrality of its institution. It can be seen from Table 3, the coefficient of the interaction term of Model 2 is positive and 
the same as the coefficient of the person center degree. This suggests that if the R\&D organization's centrality is high, the influence of its R\&D staff's centrality on innovation performance will be strengthened. This can also be confirmed from the moderating effect diagram of Figure 5. Also can be seen from Figure 5, the innovation performance of R\&D personnel will improve with the increase of its centrality.
When the center of the R\&D staff reaches a certain level, the high institutional center can further enhance the innovation performance of the R\&D personnel. Except that the node centrality is widely used in related research, this paper introduces a new variable, the clustering coefficient of the node, and finds that the clustering coefficient of the R\&D node has a significant negative influence on its innovation performance.

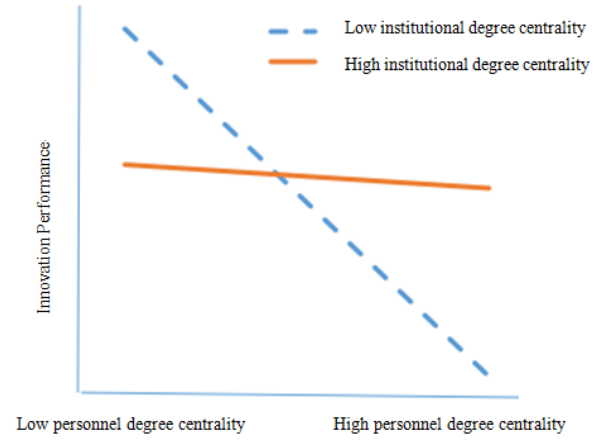

Fig.5. The moderating effect of institutional degree centrality 1

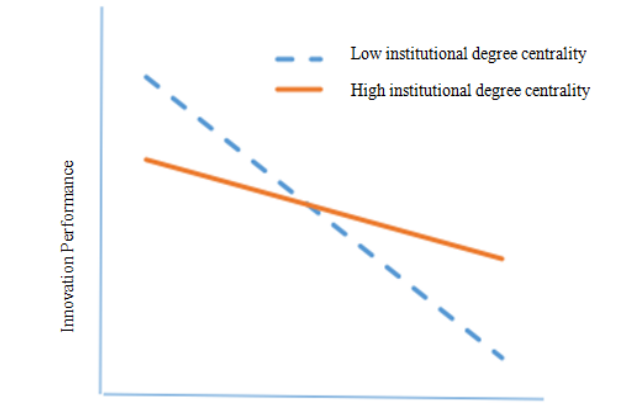

Low personnel clustering coefficient

High personnel clustering coefficient Low personnel clustering coefficient

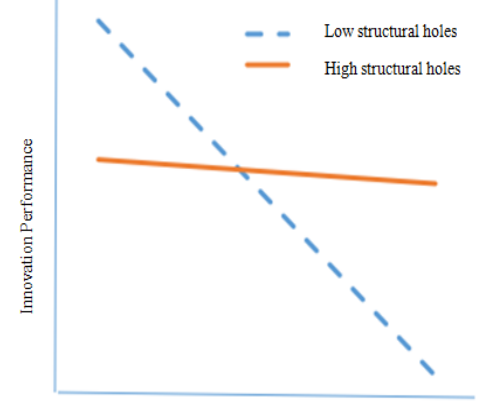

Fig.6. The moderating effect of institutional degree centrality 2
Fig.7. The moderating effect of structural holes
This effect is regulated by the centrality and structural holes in the organization where the developer's institution. It can be seen from Model 3 and Model 5 of Table 3 that the coefficient of the interaction term is opposite to the coefficient sign of the personnel clustering coefficient variable. Showing that if the R\&D institution has a high degree of centrality or high structural holes, it can weaken the negative effect of the clustering coefficient of its R\&D personnel on innovation performance. This is also confirmed in the moderating effect interaction diagrams of Figures 6 and 7. It can be seen from Figure 6 that the clustering coefficient has a significant negative effect on the employee's innovation performance; while the slope of the line corresponding to the high institution center degree is relatively flat, indicating that the high degree of institutional centrality can weaken the negative influence of clustering coefficient to some extent.

Similarly, as can be seen from Figure 7, when the structural hole position of the mechanism is low, the innovation performance of the $R \& D$ personnel with high clustering coefficient is relatively poor; and if the structural hole position of the organization is high, the positional advantage in the network is utilized. It can bring a lot of heterogeneous information and knowledge to employees, which can reduce the homogeneity impact caused by high clustering coefficient and improve the innovation performance of $R \& D$ personnel. The hypothesis 5 presented in this paper has not been supported, that is, the structural hole position in $R \& D$ institutions cannot significantly moderate the influence of the centrality of R\&D personnel on their innovation performance. The possible reason is that highly-centered employees have acquired a large amount of heterogeneous knowledge in cooperation with others, especially across organizations. Therefore, the information or knowledge acquired by the organization to take advantage of the structural hole position is not necessarily different for these employees. Qualitative, so it may not be able to strengthen the impact of its centrality on innovation performance.

\section{CONCLUSION}

This paper takes the patent data of biotechnology in Guangzhou as an example to construct a multi-level collaborative innovation network, and uses the new method of social network analysis-index stochastic graph model to analyze the status of collaborative innovation of relevant $R \& D$ institutions and $R \& D$ personnel. It was discovered that there are obvious cohesive subgroups in the R\&D network. The transfer effect of collaborative innovation between nodes in the two-level network, the lead of the core nodes and the driving action have a large room for improvement. Based on the network we built, we examined the factors that have a significant impact on the innovation performance of $R \& D$ personnel. Compared with the existing research, this paper adopts the new trend of social network research in the past two years. The perspective of multi-level network analysis not only discovers the independent variables that have a significant impact on the innovation performance of R\&D personnel, but also discovers the effect has a significant regulatory effect. That is the innovation performance of $R \& D$ personnel is not only affected by the single-level network structure, but also by the multi-level network structure. This is a result that cannot be revealed based on a single level of network analysis, and it is an important supplement to the theoretical study of innovation performance in collaborative innovation networks. We have found that the centrality of R\&D institutions can enhance the positive impact of R\&D staff's centrality on innovation performance. The centrality of the organization and the number of structural holes can weaken the negative impact of the clustering coefficient of the R\&D personnel on innovation performance. Based on this, in order to improve the innovation 
performance of its R\&D personnel, R\&D institutions should try to establish partnerships with more institutions, occupying more structural holes in the R\&D institutions network. For relevant departments, measures should be taken to build a platform for cooperation between industry, universities and research institutes. It is necessary to strengthen the synergy between R\&D personnel, as well as to strengthen exchanges between various $R \& D$ institutions, promoting the circulation of knowledge and information in the field, and to allow R\&D institutions. R\&D personnel gain heterogeneity knowledge that is conducive to innovation. At the same time, the core $R \& D$ institutions and $R \& D$ personnel in the incentive network play a leading role in improving the level of collaborative innovation in the field.

\section{ACKNOWLEDGMENT}

\section{Attached:}

Found Project: The 13th Five-Year Plan Project for the Development of Philosophy and Social Science of Guangzhou (2018GZGJ150).

\section{Author Introduction:}

Jing Li(1983-), Female, Xinhua College of Sun Yat-sen University, Birth Place HeNan, Lecturer, Master Degree, Researching in E-commerce, Data mining, Innovation Management Research.

Huimin Tang(1977-), Female, Xinhua College of Sun Yat-sen University, Birth Place ChongQing, Lecturer, Master Degree, Researching in Marketing Management and Service Innovation Management.

Harbin Tang(1982-), Male, Xinhua College of Sun Yat-sen University, Birth Place HuNan, lecturer, Master Degree, Researching in Entrepreneurship, Team Management.

\section{REFERENCES}

[1] Yiqing He, Xiaonan Qiao, Collaborative Innovation, Collaborative Innovation Network and Technology Innovation[J].Journal of Northern Nationalities University(Philosophy and Social Sciences Edition)2015,2:133-136

[2] Chaoyong Tang, Chonghuai Niu. Research on the Relationship between Collaborative Innovation Network, Talent Agglomeration Effect and Innovation Performance [J].Scientific and technological progress and actions, 2017,3:134-139.

[3] RUAN A,CHEN J. Does formal knowledge search depth benefit Chinese firms'innovation performance? Effects of network centrality, structural holes, and knowledge tacitness[J]. Asian Journal of Technology Innovation, 2017,25 (4) :1-19.

[4] PENG J etc. An empirical investigation on organizational innovation and individual creativity[J]. Information Systems and e-Business Management, 2014, 12 (3):465-489

[5] Qing Wan, Wanmin Chen, Enhua $\mathrm{Hu}$ Research on Innovation Performance of Knowledge Workers Based on Multi-level Analysis_ Considering the Influence of Double Layers of Individuals and Organizations [J].Scientific Research Management,2012,6:8-15.

[6] Lei Zhou, Wei Yang, Yufeng Zhang. Breakthrough Innovation and Identification Framework Research Based on Patent Mining [J]. Intelligence Theory and Practice,2016,9:73-76.

[7] BAUM J, COWAN R, JONARD N. Network-Independent Partner Selection and the Evolution of Innovation Networks. Management Science,2010,11: 2094-2110.
[8] HARRIS J, Guancan Yang. Introduction to Exponential Stochastic Graph Model[M].ShangHi: GeZhi Press 2016:83-84.

[9] Xuan Zhang, GuoShun Wang. The influence of network centrality and knowledge innovation ability on innovation performance [J].Economic Issues, 2013,8:92-96.

[10] GUAN $J$ etc. The impact of multilevel networks on innovation[J].Research Policy, 2015,44: 545-559.

[11] Yong Cao etc. The Influence of Heterogeneous Knowledge on Enterprise Innovation Performance: Theoretical Review and Prospects[J].Science and Technology Management Research $2016,36(2): 168-171$.

[12] Xuemei Xie, Leilei Zuo, The Characteristics of Enterprise Collaborative Innovation Network and Innovation Performance: A Study of the Mediating Effect Based on Knowledge Absorptive Capability[J] Nankai University Management Comment,2013,3:47-56.

[13] EISINGERICH AB,BELL SJ,TRACEY P. How can clusters sustain performance? The role of network strength, network openness, and environmental uncertainty[J]. Research Policy, 2010,39 (2), 239-253.

[14] MASON W, WATTS DJ. Collaborative learning in networks[J].Proceedings of the National Academy of Science of the United States of America, 2012 , 109 (3) :764-769.

[15] Xiaoming Sun etc. The influence of structural hole changes on the innovation ability of enterprises in the patent cooperation network of developers[J].Science and Technology Progress and Action,2018,2:115-122.

[16] KIJKUIT B, VAN EJ. With a little help from our colleagues: a longitudinal study of social networks for innovation[J].Organization Studies, 2010, 31 (4) :451-479.

[17] R.S Burt. Structural holes and good ideas[J].American Journal of Sociology,2004,110 (2):349-399.

[18] SHIPILOV A. Firm scope experience, historic multimarket contact with partners, centrality, and the relationship between structural holes and performance.Org. Sci,2009, 20 (1):85-106. 